\title{
Ueber einige geometrische Sätze.
}

(Von Herrn von Staudt zu Erlangen.)

1. Das Product aus dem Inhalte $\Pi$ eines Tetraeders $\boldsymbol{A B C D}$ in den Halbmesser $r$ der um dasselbe beschriebenen Kugelfläche ist durch die drei Producte DA.BC, DB. AC, DC.AB bestimmt. Bezeichnet man diese Producte durch $a, b, c$, die Hälfte ihrer Summe durch $s$ und die Quadratwurzel aus dem Producte $s(s-a)(s-b)(s-c)$ durch $\omega$, so ist $\left.6 \Pi \cdot r=\omega^{*}\right)$.

Wenn nämlich die eine von zwei zu einander parallelen Ebenen, welche den Abstand $h$ von einander haben, die erwähnte Kugelfläche im Punkte $\boldsymbol{D}$ berührt, so schneidet die andere die Geraden $\boldsymbol{D A}, \boldsymbol{D B}, \boldsymbol{D C}$ in drei Punkten $A_{1}, B_{1}, C_{1}$, so dafs

$$
\boldsymbol{D A} \cdot D A_{1}=D B \cdot D B_{1}=D C \cdot D C_{1}=2 r h
$$

ist. Wenn man ferner den Inhalt des Dreiecks $A_{1} B_{1} C_{1}$ durch $\triangle_{1}$, den Inhalt des Tetraeders $A_{1} B_{1} C_{1} D$ durch $\Pi_{1}$, das Product $D A . D B . D C$ durch $p$ und das Product $\boldsymbol{D} \boldsymbol{A}_{1}, D B_{1}, D C_{1}$ durch $p_{1}$ bezeichnet, so ist:

$$
3 \Pi_{1}=\triangle_{1} h, \Pi p_{1}=\Pi_{1} p, p p_{1}=8 r^{3} h^{3} \text {. }
$$

Da nun wegen Aehnlichkeit der Dreiecke $D B C, D C_{1} B_{1}$ die Producte $D B . B_{1} C_{1}$, $D C_{1} . B C$ einander gleich sind, so folgt, wenn man beide mit $\boldsymbol{D A} . \boldsymbol{D C}$ multiplicirt, dafs $p . B_{1} C_{1}=2 r h a$ ist. Da ebenso p. $A_{1} C_{1}=2 r h b$ und p. $A_{1} B_{1}$ $=2 r h c$, so ist $p^{2} \triangle_{1}=4 r^{2} h^{2} \omega$, demnach $3 \Pi_{1} p^{2}=4 r^{2} h^{3} \omega$, also auch $3 \Pi p p_{1}=4 r^{2} h^{3} \omega$ und mithin $6 \Pi r=\omega$.

Ist $h=\frac{p}{4 r^{2}}$, folglich $2 r h=\frac{p}{2 r}$, so ist

$$
\begin{array}{ccc}
2 r \cdot D A_{1}=D B \cdot D C, & 2 r \cdot D B_{1}=D A \cdot D C, & 2 r \cdot D C_{1}=D A \cdot D B, \\
2 r . B_{1} C_{1}=a, \quad & 2 r \cdot A_{1} C_{1}=b, & 2 r \cdot A_{1} B_{1}=c, \\
4 r^{2} \triangle_{1}=\omega & \text { und mithin } \Pi=\frac{2}{3} r \triangle_{1} .
\end{array}
$$

2. Ist $\triangle$ der Inhalt des Dreiecks $\boldsymbol{A B C}, \boldsymbol{u}$ aber das Product aus der kleinsten $\boldsymbol{D P}$ und gröfsten $\boldsymbol{D Q}$ von allen Strecken, welche von dem Punkte $\boldsymbol{D}$ an den Umfang $\boldsymbol{K}$ des um das Dreieck $\boldsymbol{A B C}$ beschriebenen Kreises gezogen werden können, so ist $\Delta u=\omega$, wo $\omega$ den vorigen Ausdruck bedeutet.

Wenn nämlich der Punkt $\boldsymbol{D}$ aufserhalb der Ebene $\boldsymbol{A B C}$ liegt und von derselben den Abstand $d$ hat, so ist nach dem vorigen Satze $2 \Delta d r=\omega$.

*) Vergleiche Crelle (malhematische Aufsätze I, p. 117) und Joachimsthal Bd.40, p. 33 dieses Journals. 
Da aber $\boldsymbol{r}$ auch der Halbmesser des um das Dreieck $\boldsymbol{D P Q}$ beschriebenen Kreises ist, so ist nach einem bekannten Satze $2 d \boldsymbol{r}=\boldsymbol{D P} . \boldsymbol{D Q}$ und folglich $\triangle \boldsymbol{u}=\boldsymbol{\omega}$. Liegt der Punkt $\boldsymbol{D}$ in der Ebene $\boldsymbol{A B C}$ aber aufserhalb der Kreislinie $\boldsymbol{K}$, so seien $\boldsymbol{A}_{1}, \boldsymbol{B}_{1}, \boldsymbol{C}_{1}$ diejenigen Punkte dieser Linie, welche aus den Punkten $\boldsymbol{A}, \boldsymbol{B}, \boldsymbol{C}$ durch die Geraden $\boldsymbol{A D}, \boldsymbol{B D}, \boldsymbol{C D}$ projicirt werden. Wenn man nun wieder das Product $\boldsymbol{D A} . \boldsymbol{D B} . \boldsymbol{D C}$ durch $\boldsymbol{p}$, das Product $\boldsymbol{D} \boldsymbol{A}_{1}, D B_{1}, D C_{1}$ durch $p_{1}$ und den Inhalt des Dreiecks $\boldsymbol{A}_{1} \boldsymbol{B}_{1} \boldsymbol{C}_{1}$ durch $\triangle_{1}$ bezeichnet, so hat man die Gleichungen:

$$
\begin{gathered}
\text { DA.DA } A_{1}=D B \cdot D B_{1}=D C \cdot D C_{1}=u, \\
p . B_{1} C_{1}=u a, p \cdot A_{1} C_{1}=u b, p \cdot A_{1} B_{1}=u c,
\end{gathered}
$$

woraus hervorgeht, dafs $p^{2} \triangle_{1}=u^{2} \omega$ ist. Weil aber die Dreiecke $\boldsymbol{A B C}$, $\boldsymbol{A}_{1} \boldsymbol{B}_{1} \boldsymbol{C}_{1}$ in einen und denselben Kreis beschrieben sind, so ist

$$
\frac{\triangle}{\triangle_{1}}=\frac{A B}{A_{1} B_{1}} \cdot \frac{A C}{A_{1} C_{1}} \cdot \frac{B C}{B_{1} C_{1}}=\frac{D A}{D B_{1}} \cdot \frac{D C}{D A_{1}} \cdot \frac{D B}{D C_{1}}=\frac{p}{p_{1}}=\frac{p^{2}}{u^{3}},
$$

mithin $\triangle u=\triangle_{1} \cdot \frac{p^{2}}{u^{2}}=\omega$.

Wenn endlich der Punkt $\boldsymbol{D}$ in der Linie $\boldsymbol{K}$ liegt, so fällt $\boldsymbol{P}$ mit $\boldsymbol{D}$ zusammen, daher in diesem Falle $\boldsymbol{D P}$ und also auch $\boldsymbol{u}=0$. In diesem Falle ist aber das gröfste der drei Producte $a, b, c$ der Summe der beiden übrigen gleich und daher auch $\omega=0$. Bemerkt wird noch, dafs, wenn der Punkt $\boldsymbol{D}$ von allen Punkten der Linie $\boldsymbol{K}$ gleichweit absteht, unter $\boldsymbol{u}$ das Quadrat dieses Abstandes zu verstehen ist.

3. Wenn $m$ das geometrische Mitlel zwischen der kleinsten und gröfsten von allen Strecken ist, welche aus dem Punkte $\boldsymbol{D}$ an eine durch die drei Punkte $\boldsymbol{A}, \boldsymbol{B}, \boldsymbol{C}$, aber nicht durch den Punkt $\boldsymbol{D}$ gehende Kreislinie gezogen werden können, so hat das Dreieck, dessen Seiten die Längen $\frac{\boldsymbol{D A} . \boldsymbol{B C}}{m}$, $\frac{D B \cdot A C}{m}, \frac{D C \cdot A B}{m}$ haben, mit dem Dreiecke $A B C$ gleichen Inhalt. Ist $A B C$ ein gleichseitiges Dreieck, so hat das gleichseitige Dreieck, dessen Seite $m$ ist, mit dem Dreiecke gleichen Inhalt, dessen Seiten den Strecken $D A, D B$, $\boldsymbol{D C}$ gleich sind.

4. Entsprechen den Punkten $\boldsymbol{A}, \boldsymbol{B}, \boldsymbol{C}, \boldsymbol{D}$ der einen von zwei Kugelflächen, welche projectivisch auf einander bezogen sind, die Punkte $A_{1}, B_{1}$, $C_{1}, D_{1}$ der anderen, so ist, wie sich leicht nachweisen läfst:

$$
D A . B C: D B . A C: D C . A B=D_{1} A_{1}, B_{1} C_{1}: D_{1} B_{1} \cdot A_{1} C_{1}: D_{1} C_{1} \cdot A_{1} B_{1}
$$

